\title{
Ovarian carcinoma: pathology review with an emphasis in their molecular characteristics
}

\author{
Leonardo S. Lino-Silva \\ Surgical pathology, Instituto Nacional de Cancerología, Mexico City, Mexico \\ Correspondence to: Leonardo S. Lino-Silva, MD, M.Ed. Anatomic Pathology Professor, Gastrointestinal Pathology Division, Instituto Nacional de \\ Cancerología de México (Mexico’s National Cancer Institute), Av. San Fernando \# 22, Sección XVI, Tlalpan, CP 14080 Mexico City, Mexico. \\ Email: saul.lino.sil@gmail.com.
}

\begin{abstract}
Ovarian carcinoma is highly aggressive and difficult to treat neoplasm, which is usually detected in advanced stages where most patients recur. Extensive investigation about several treatment modalities has been performed but this neoplasm has poor benefits from such treatments including targeted therapy. Recent data have begun to highlight the histological and molecular heterogeneity of these tumors defining this neoplasm, not as a single disease but a group of heterogeneous histological subtypes with important differences in terms of genetics, morphology, oncogenesis, prognosis, chemosensitivity and especially molecular characteristics that are likely to be targets of new molecules. In general, high-grade serous carcinomas are characterized by great genomic instability and frequent amplifications and deletions; lowgrade ovarian neoplasms are genomically stable. On this phytopathogenic basis, recent findings suggest a dual model of carcinogenesis consisting of two large groups named types I and II. Type I cancers (serous, mucinous, and low-grade endometrioid) commonly arise from well-described, genetically stable precursor lesions (usually borderline tumors); manifests as large adnexal masses with the early-stage disease, and they have a good overall prognosis. In contrast, type II carcinomas (serous, high-grade endometrioid, mixed, and undifferentiated carcinomas) originate de novo from the adnexal epithelium, often demonstrate chromosomal instability, and have aggressive biological behavior. Surprisingly, most of the genomic abnormalities detected encode known oncogenic proteins for which there is targeted therapy. Then, there is a real potential for personalized medicine adapted to the molecular portrait of tumors. In this review, I synthesize the histology and molecular pathology of ovarian carcinomas and possible strategies to reach targeted therapy.
\end{abstract}

Keywords: Ovarian carcinoma (OCA); molecular pathology; histopathology

Submitted Jan 20, 2020. Accepted for publication May 08, 2020.

doi: $10.21037 / \mathrm{cco}-20-31$

View this article at: http://dx.doi.org/10.21037/cco-20-31

\section{Introduction}

Ovarian cancer (OC) is the main cause of death from gynecological cancer because most is at an advanced stage at diagnosis. Despite the initial chemosensitivity, relapses are frequent and the median overall survival is less than 5 years. In the last decades, the prognosis of women with OC has not improved. The initial treatment of these cancers is based on a complete surgery combined with chemotherapy based on platinum. One of the reasons that could explain the still disappointing results for these cancers is probably because of management is homogeneous, regardless of the morphology or histologic subtype. In fact, the progress made for melanoma, lung cancer, and breast cancer can be explained because of treatments are increasingly adapted to the histology or genetics of the neoplasms. The first success of a truly targeted therapy for tumor abnormality was hormonal therapy in breast cancers that express estrogen receptors. Since then, many therapies adapted to tumor biology have been approved: trastuzumab for breast cancers that overexpress the HER2 receptor, gefitinib and erlotinib, 
or crizotinib in mutated $E G F R$ or altered $A L K$ lung cancers, respectively, and vemurafenib in mutated $B R A F$ melanomas. In comparison, the treatment of OC is completely uniform. Recent studies have contributed to the characterization of their histological subtypes and have highlighted the heterogeneity of these tumors, with significant histological, genomic, prognostic and chemosensitivity differences. Unfortunately, this better understanding of carcinogenesis and ovarian molecular biology has not yet translated into concrete therapeutic advances for our patients. The vast majority of trials of targeted therapies in OCA are negative (1). Bevacizumab is the only innovative therapy with marketing authorization for this indication, but a predictive biomarker has not yet been identified to select patients who can benefit from this antiangiogenic agent. In this article, I propose to synthesize data from the literature on genetics of OC, morphology and basis for specific strategies in this cancer. Proposals for optimizing translational research (quality sequential tumor samples, potential for liquid biopsies, new clinical trial models), accelerating the development of specific treatments and thus improving the personalized care of our patients will be considered.

\section{The basis}

The vast majority of OC are epithelial ( $75 \%$ to $80 \%$ ). Non-epithelial tumors are less common and include germline tumors, sex cord tumors and other rare tumors, such as small cell cancers. Non-epithelial OC tends to develop in younger women, is diagnosed at an earlier stage and generally has a better prognosis. The rest of this article will focus on ovarian carcinomas (OCAs). OCAs have typically been described as high-grade tumors, with serous histology, which occurs in patients $>60$ years and diagnosed in an advanced stage ( $75 \%$ in stage IIIIV), are poorly differentiated, and they rarely express estrogen receptors. The initial response to chemotherapy is sometimes dramatic, but it is often short-lived. It is evident today that these generalities apply especially to high-grade serous OCAs that in fact constitute only a proportion of OCAs. During the 1950s and 1960s, morphological and histological studies of OCAs identified less common histological subtypes, such as mucinous, clear cell, endometrioid, or low-grade serous carcinoma. These data led to a morphological reclassification of the OCAs by the World Health Organization (1973). More recently, a dualistic model widely adopted by the scientific community divides OCAs into two groups according to their grade and morphology: type I includes low-grade serous tumors, mucinous tumors, endometrioid tumors, clear cell tumors, and transitional tumors; type II includes high-grade serous OCAs, carcinosarcomas and undifferentiated carcinomas (2). Unfortunately, despite a better characterization of these various histological types, OCAs are still treated as a single disease. As will be developed in the following paragraphs, today it has become clear that these histological distinctions have important consequences in the management of each subtype because they correspond to significant differences in terms of tumor carcinogenesis. clinical aggressiveness, chemosensitivity and, above all, genomic abnormalities likely to be the target of new molecules $(3,4)$.

\section{The heterogeneity of OCAs}

\section{Type I OCAs}

Type I OCAs are typically low grade, better differentiated, genomically stable, characterized by the absence of $\mathrm{p} 53$ mutation. They appear at an earlier stage, often limited to the ovary (stage I), where surgical excision allows a higher cure rate than for types II. Despite this chemotherapy with platinum salts seems less effective in recurrence $(15 \%$ to $30 \%$ of response rate compared to $75 \%$ in high grades). Most of these malignant tumors progress gradually from serous or mucinous cystadenomas, or endometriosis in the case of endometrioid or clear cell carcinomas (CCCs). The different subtypes are very heterogeneous and have few common histological characteristics; their morphologies are often similar to other primitives such as digestive or urological tumors (5). Therefore, the low-grade mucinous cancers present a glandular differentiation of the intestinal type $(80 \%)$ or endocervical $(20 \%)$. Given its modest response to platinum salts $(25 \%)$ and its morphological similarity to intestinal cancers, a randomized clinical trial comparing the standard protocol for OCAs (carboplatin + paclitaxel) with that of digestive tumors (capecitabine + oxaliplatin) with or without bevacizumab is in progress. CCCs respond poorly to a combination of platinum and paclitaxel (response of 15\%). They are morphologically similar to clear cell kidney cancer, and genomic expression studies have suggested an angiogenic signature similar to that tumor (5). As a result, a study is being conducted to evaluate sunitinib antiangiogenic in CCC. These studies are the first examples of histo-specific trials and a first step towards the treatment of OCAs adapted to tumor biology. 


\section{Type II OCAs}

Type II OCAs, whose prototype is high-grade serous carcinoma, also includes carcinosarcomas (or mixed Muller tumors) and undifferentiated carcinomas. Some also suggest the inclusion of high-grade endometrioid OCAs, although the existence of this distinct histological entity remains controversial. Carcinosarcomas are biphasic, epithelial (most often serous or endometrioid, but always grade 3 ) and mesenchymal tumors. Given its degree and aggressiveness, and despite its sarcomatous component, they are now included among epithelial ovarian tumors type II. Highgrade OCAs are diagnosed at an advanced stage and most require surgical excision and chemotherapy with platinum salts. Despite a response rate to a combination of platinumpaclitaxel of the order of $75 \%$, relapses are almost inevitable and explain a 5 -year survival rate of the order of $30 \%$. They grow from surface cells of the ovary or salpinx, and some authors suggest an origin not from pre-invasive lesions, but develop de novo associated with spontaneous mutations of p53 (2). An inactivating mutation of the p53 suppressor gene is practically ubiquitous in OCAs type II (>95\%) (6). These tumors are characterized not only by a high proliferation rate but also by great genetic instability, due to significant deficits in their DNA repair pathways. A $10-15 \%$ of high-grade serous OCAs have a constitutional mutation of BRCA1 or BRCA2 (breast cancer 1/2). These mutations result in the loss of key proteins involved in genome stability and repair of damaged DNA, particularly in the homologous recombination $(\mathrm{RH})$ repair pathway of double chain breaks generated by platinum salts. $B R C A$ mutations explain the genetic instability and sensitivity to platinum salts observed in patients with mutated $B R C A$ OCAs (3). Since, despite the excellent initial response to platinum salts, relapses are almost inevitable and often chemoresistant, many efforts have been made to identify strategies to restore chemosensitivity. The DNA repair deficits identified in some high-grade OCAs represent a potentially exploitable Achilles heel for therapeutic purposes. In the absence of functional BRCA1 or BRCA2 proteins, DNA breaks induced by plaques are repaired by an additional signaling pathway, the repair of base cleavage. Poly-ADP-ribose polymerase (PARP) is a coordinating enzyme for this repair pathway and PARP inhibitors have shown encouraging results in mutated BRCA OCAs (4).

\section{The molecular profile of ovary carcinomas}

\section{Molecular alterations in high-grade serous OCAs type II}

In addition to almost universal p53 mutations and functional $B R C A$ mutations in $15 \%$ of cases, high-grade OCAs have long been described as tumors that do not cause difficult driver mutation to develop targeted treatments for these tumors However, recent results suggest that high-grade tumors may have a large number of molecular alterations. Given the rarity of other high-grade histological types (undifferentiated OCAs or carcinosarcomas), the molecular studies included in this chapter are limited to high-grade serous carcinomas.

\section{Mutations in high-grade serous carcinomas}

When sequencing exomes, The Cancer Genome Atlas (TCGA) confirmed the presence of $p 53$ mutations in $96 \%$ and constitutional BRCA1 or BRCA2 in $15 \%$ of 300 high-grade serous OCAs (6). Other mutations were rare. Inactivating mutations of neurofibromatosis-1 (NF1) or retinoblastoma-1 (RB1) suppressor genes were found in $4 \%$ and $2 \%$ of tumors, and somatic mutations of BRCA1 or $B R C A 2$ in $6 \%$. Known oncogenes activating mutations are rarer, such as PIK3CA, KRAS, BRAF, PTEN, HER2, $E G F R, K I T$, and others, each found in less than $1 \%$ to $2 \%$ of the 300 tumors analyzed. A similar study using another technology (OncoMap and Sequenom) showed a slightly higher mutation rate among 200 high-grade OCAs with the following alterations EGFR (10\%), KRAS (5\%), PDGFR (5 \%), KIT (3\%), PIK3CA (3\%), NRAS (2.5\%) and $B R A F(1 \%)(7)$.

\section{Amplifications and deletions in high-grade serous carcinoma}

In comparison, the gains and losses of the chromosomal portions are much more frequent, which is not surprising given the instability in the same TCGA project, a study of segmental chromosomal alterations in the 300 OCAs was performed (high grade and showed significant amplification and loss). These genetic abnormalities seem much more frequent than mutations, for example, an amplification of $M A P K 15, M Y C$ or CCNE1 were detectable in $28 \%, 27 \%$ and $23 \%$ of tumors, respectively. It is also notable that 22 of 
the most amplified genes encode known oncogenic proteins for which a targeted therapy exists or is being developed.

\section{Specific genetic alterations in high-grade serous carcinoma}

Amplifications of membrane receptors with tyrosine kinase activity (EGFR, HER2, HER3 or IGF1R) are detected in $15 \%$ of these tumors, abnormalities in the GTPase pathway, RAS [NF1, KRAS, NRAS, mitogen-activated protein kinase (MAPK)] in 35\% to $40 \%$, and abnormalities in the phosphoinositide-3 kinase pathway (PI3K) (PIK3CA, PTEN, AKT1/2, RAPTOR/RICTOR) in almost 40\%. An American Society of Clinical Oncology (ASCO) presentation described objective molecular abnormalities only in $20 \%$ of OCAs (for most high-grade serous carcinomas) (8). It should be noted that, under this project, directed research was limited to 15 genes using the $\mathrm{SNaPshot} \mathrm{kit} \mathrm{in} 83$ paraffin embedded tumors. The number of tumors included, the kit used and the use of paraffin tumor samples could explain the discordant results obtained by this group. Finally, only one p53 mutation was detected in $19 \%$ of the tumors, which also makes it possible to question the validity of the conclusions of this study. Taken individually, genomic alterations are rare, which means that to be useful and informative, the profile of high-grade OCAs will require a systematic and thorough search of each tumor to identify significant abnormalities. The use of broadband technologies such as next-generation sequencing seems inevitable.

\section{DNA repair}

Recent extensive studies suggest that $50 \%$ of highgrade serous OCAs may be deficient in the hormonal receptors pathway. In addition to the constitutional mutations of BRCA1 and BRCA2, somatic mutations have also been discovered in $5 \%$ to $6 \%$ of high-grade serous OCAs. Hypermethylation of the BRCA1/2 promoter is described in $10 \%$ of these tumors (6). These abnormalities are mutually exclusive, which means that $30 \%$ of high-grade OCAs may have a loss of BRCA function. Beyond BRCA, other proteins ensure the proper functioning of hormonal receptors: EMSY, a BRCA suppressor gene, is amplified in 5\% to $17 \%$ of high-grade ovarian tumors, $R A D 51$, an important effector, is lost in $3 \%$ to $5 \%$, and other genes involved in hormonal receptors pathway (ATR, ATM and Fanconi genes) are mutated in $8 \%$. It is probable that genetic instability also provides plasticity that facilitates the appearance of drug-resistant subclones and allows tumor progression. The identification and molecular characterization after treatment of these high-grade tumors, enriched with resistant clones, could allow us to better understand how these tumors adapt to chemotherapy and identify possible therapeutic objectives for serous type II carcinomas.

\section{The molecular profile of type I OCAs}

Each of the subtypes of type I tumors is rarer, the data related to their molecular abnormalities are less numerous and, in particular, less than complete genomic sequencing. Rather, searches for specific mutations were carried out. These studies, even modest, still allowed a useful characterization of the most frequent alterations.

\section{Low-grade serous carcinomas}

These tumors have frequent dysfunctions in the $R A S$ signaling network. Activating mutations of KRAS $(20 \%$ to $40 \%$ ) and BRAF (5\% to $10 \%$ ) have been identified (2). They are even more frequent in pre-invasive lesions, such as serous cystadenomas or serous borderline tumors, suggesting a loss of KRAS and $B R A F$ mutations during tumor progression (9). A HER2 mutation has also been described in $9 \%$ of low-grade serous carcinomas (10).

\section{Clear cell carcinoma}

Despite their inclusion among type I carcinomas, known as low-grade carcinomas, these tumors tend to be more aggressive with a response to chemotherapy of the order of $10 \%$ to $15 \%$. One of the most common abnormalities in these tumors is an inactivating mutation in the ARID1A suppressor gene (11). ARID1A is a chromatin regulator that is mutated in $50 \%$ of clear cell tumors. This mutation is also found in endometriosis samples and in 30\% of low-grade endometrioid carcinomas, which suggests that this anomaly occurs early in oncogenesis and confirms the link between these two types of cancer with endometriosis. In CCC, there is often an over-activation of the $P I 3 K$ signaling pathway: $30 \%$ to $35 \%$ have a mutation in the p110 catalytic subunit of PI3K (PIK3CA), and 20\% a loss of PTEN suppressor gene (12). Also, an array comparative genomic hybridisation $(\mathrm{aCGH})$ analysis of 50 tumors revealed that $14 \%$ of the CCC demonstrated amplification of HER 2 and confirmed 
the overexpression of HER2 by immunohistochemistry (13). c-Met, a receptor with tyrosine kinase activity that stimulates the Ras, PI3K and catenin pathways, is amplified in $15 \%$ to $20 \%$ of the CCC (12). Its ligand, hepatocyte growth factor (HGF) is also overexpressed in a proportion. Given the morphological relationship of CCC with clear cell renal tumors, it is not surprising that overexpression of hypoxiainduced alpha-factor (HIF1) is found in some CCC (12).

\section{Endometrioid carcinomas}

Despite a possible common origin with CCC (endometriosis), low-grade endometrioid carcinomas have a different molecular picture than CCC. They are characterized by an activation of the -catenin signaling pathway due to a CTNNB1 activating mutation found in $40 \%$ of cases (14). They are also the only ovarian tumors with frequent PTEN mutations. They also have PIK3CA mutations and loss of genes by PTEN. In summary, Most of endometrioid tumors express the activation of the catenin pathway (40\%) or PI3K (60\% to $80 \%)$.

\section{Mucinous tumors}

These tumors are rare $(<5 \%)$ and RNA expression studies have shown a similarity with colorectal carcinomas. Mucinous OCAs also share other characteristics with digestive mucinous tumors, such as microsatellite instability (MSI) (20\%) or KRAS mutations (30\% to 50\%) (15). The overexpression of HER2 is found in $15 \%$.

\section{Transitional tumors (Brenner tumors)}

These tumors are the rarest. They share histological characteristics with urothelial tumors. Therefore, the diagnosis of transitional OCAs requires first to exclude a primitive urothelium, but there is no data available on its molecular profile.

\section{Biological justification of personalized medicine for ovary cancer}

Given the detectable genomic alterations in the OCAs and the number of new molecules, approved or in development, directed at these same anomalies, it is reasonable to expect that taking the charge adapted to tumor biology is an attainable goal in the near future. Below are some particularly promising approaches based on the molecular biology of OCAs.

\section{DNA repair capacity}

PARP inhibitors have shown encouraging results in mutated BRCA OCAs. The recent demonstration that $50 \%$ of high-grade cancers potentially have a deficit in the heart rate pathway suggests that the benefit of these inhibitors could be explored in a trial that recruits specifically selected patients based on a screening test. Functional $\mathrm{RH}$ (constitutional or somatic $B R C A$ mutations, $B R C A$ promoter hypermethylation, $E M S Y$ amplification, loss of RAD 51, ATM/ATR mutation). In addition, 20\% of highgrade serous tumors are refractory to platinum salts. The predictive markers that allow us to identify this drugresistant subgroup would help avoid exposing these patients to toxic and ineffective treatments. Data from other solid tumors suggest that DNA repair markers, such as ERCC1, can predict platinum sensitivity (16). It has also been shown that other key proteins involved in DNA repair correlate with chemosensitivity (17). A panel of markers representing the functionality of DNA repair pathways could help identify high-grade platinum-resistant ovarian tumors and offer these patients another chemotherapy or experimental treatment.

\section{Angiogenesis}

Without a doubt, the most promising strategy for OCA is the targeting of angiogenesis. Marketing authorization has been assigned to the bevacizumab antibody combined with chemotherapy and then in maintenance, a significant improvement in progression-free survival has been demonstrated in several phase III trials (18). But it is notable that, unlike most other solid tumors, where the benefit of bevacizumab requires an association with chemotherapy, $15 \%$ to $20 \%$ of patients treated in the first phase II studies showed an objective tumor response to bevacizumab alone (19). Unfortunately, to date, no biomarker has been validated for anti-vascular treatments. The predictive value of a $V E G F A$ amplification (present in $5 \%$ of the high-grade serous carcinoma), or overexpression of HIF 1 described in certain CCC deserves to be explored. Meanwhile, according to an analogy with clear cell kidney cancer, a test of the VEGFR inhibitor, sunitinib, is being performed on the CCC. 


\section{PI3K}

Forty percent of high-grade carcinomas and half of lowgrade endometrioid carcinomas have constitutive activation of the PI3K/Akt/mTOR pathway (target of rapamycin). This intracellular signal pathway plays a key role in the control of the cell cycle, and the inhibitors are available for multiple effectors in this cascade (selective inhibitors of mTOR, PI3K, Akt or mixed inhibitors PI3K/mTOR or Akt/ mTOR). The results of the first trial of an $m T O R$ inhibitor in OCAs were disappointing with a $9 \%$ of tumor response and the researchers concluded that there was no reason to continue with a phase III study (19). Undoubtedly, these negative results may be due because of the recruitment of an unselected population. In fact, MD Anderson researchers recently reported that $P I 3 K$ mutations predict a response to $P I 3 K$ pathway inhibitors in gynecological cancers (including 56 OCAs) with a $30 \%$ of response compared to only $10 \%$ among not mutated (20). However, the predictive value of $P I 3 K$ pathway biomarkers remains controversial with data on endometrial cancer that show no association between PI3K mutations or loss of PTEN and tumor response. Another hypothesis to explain the limited activity of temsirolimus comes from observations on resistance mechanisms. The complexity and redundancy of the signaling network, as well as the existence of feedback loops through IGF1R or Akt, allow tumor cells to escape selective $m T O R$ inhibition. An effective and sustained antitumor effect may require the use of mixed inhibitors or a combination with a MAPK or IGF1R inhibitor.

\section{$R A S$}

This route directly controls the cascade of the Raf/MAPK/ Erk effector and indirectly the $P I 3 K$ route. High-grade carcinomas show amplification of KRAS in $11 \%$ of cases, loss of the NF1 suppressor in $4 \%$ and mutations of NRAS/ $K R A S$ or $B R A F$ in $6 \%$ to $7 \%$. As for low-grade serous and mucinous carcinomas, an activating mutation is detected in $30 \%$ to $60 \%$ of cases. Therefore, OCAs could be rationally selected on the basis of genomic expression for $B R A F$ inhibitors, or for a subsequent effector inhibitor, Mek. These mutations also appear to cause resistance to PI3K inhibitors, and tumors with abnormalities of PI $3 K$ and $K R A S$ are particularly susceptible to coordinated inhibition of Mek and PI3K in vitro (21). Simultaneous mutations of PI3K and KRAS are described in patients with OCAs (20). Therefore, it is worth exploring a combination of Mek and
PI3K/mTOR inhibitors in this subgroup.

\section{Met/HGF}

Preliminary data indicate encouraging activity of Met inhibitors in amplified MET lung cancer. MET amplification is observed in a proportion (20\%) of CCCs. A phase II trial of cabozantinib, an inhibitor of VEGFR2 and Met, showed an encouraging tumor response of $25 \%$ in OCAs (22).

\section{Membrane receptors}

Several studies have been conducted with EGFR inhibitors (erlotinib, gefitinib) because of most OCAs express the EGFR protein; however, all were negative, including a phase III clinical trial. These trials were probably limited by the inclusion of an unselected population. It is notable that the only objective response to gefitinib, reported in a phase II trial, was observed in OCA with an EGFR mutation (23). Although taken individually, membrane receptor abnormalities are rare, an EGFR mutation is detectable in $9 \%$ of high-grade serous carcinomas, amplification of HER2/HER3 in 7\% of highgrade adenocarcinomas and a mutation or amplification of HER 2 in $30 \%$ to $40 \%$ of type I OCAs. Given the activity of inhibitors (erlotinib-gefitinib, trastuzumab, trastuzumabDM1, lapatinib, and pertuzumab) in bronchial, mammary or gastric carcinomas with a proven mutation or amplification, these inhibitors may also have antitumor activity in certain OCAs, choosing the molecule according to the genetic abnormality detected. A trial with pertuzumab also showed an improvement in tumor response and progression-free survival in OCAs, especially in the subgroup with low expression of HER3 mRNA (24). A phase II pertuzumab trial is being conducted in this subgroup.

\section{Folate receptor (RF)}

$\mathrm{RF}$ is expressed in $70 \%$ of OCAs at the time of diagnosis and in $82 \%$ during a relapse but is rarely found in healthy tissue. EC145, a combination of folic acid and diacetyl vinblastine, has been evaluated in phase I and phase II trials and has shown an antitumor effect in women with platinum-resistant OCA (25). A randomized phase III study comparing Caelix ${ }^{\circledR}$ alone with Caelix ${ }^{\circledR}$ in combination with EC145 is underway. All participants will benefit from a nuclear radiology diagnostic test for RF to determine the predictive value of this test to select patients who express 
the molecular objective of EC145.

\section{NOTCH}

The NOTCH signaling pathway coordinates the transcription of genes involved in oncogenesis and the maintenance of stem cells. The amplification of NOTCH3 is described in $10 \%$ to $20 \%$ of OCAs and the TCGA has described the activation of the NOTCH signaling pathway in $20 \%$ of the 300 high-grade tumors analyzed. Several phase I trials evaluating the efficacy and toxicity of a NOTCH inhibitor alone or in combination with other targeted therapies are ongoing in OCAs.

\section{Anti-mullerian bormone receptor 2(AMHR2)}

The anti-mullerian hormone (AMH), also known as the Mullerian inhibitory substance (MIS), is a counterpart of the transforming growth factor beta (TGF-beta). In embryonic development, AMH regulates the sexual differentiation of the genitals. Regarding carcinogenicity, the antimullerian hormone receptor 2 (AMHR2) is overexpressed in $50 \%$ of OCA and in $96 \%$ of granulosa ovarian tumors. Targeted treatment of AMHR2 has a pro-apoptotic effect on OCA cells and increases the efficacy of cisplatin in vitro (26). Humanized antibodies against AMHR2 are still in a preclinical stage of development, but offer another illustration of a potential therapy adapted to the tumor's molecular profile.

\section{The incorporation of molecular biology in clinical trials for ovary cancer}

Despite the real progress in molecular characterization of OCAs and solid preclinical data, the vast majority of clinical trials evaluating new molecules are negative (1). The series of GOG 170 trials evaluating the effectiveness of several targeted therapies (gefitinib, lapatinib, vorinostat, imatinib ...) in OCAs reported responses of less than $15 \%$. It is important to note that these trials were phase II, an arm, evaluating the activity of monotherapy in an unselected population. Undoubtedly, several factors contribute to explaining these disappointing results: (I) the inclusion of an unselected population; (II) a non-random design that makes it difficult to estimate the effectiveness of the treatment; (III) possibly, the fact that certain molecules should be used in combination with other targeted therapies to elicit a sustained antitumor response. Solutions can be proposed to optimize the incorporation of OCA biology in clinical research and facilitate the development of innovative molecules for this disease.

\section{Access to quality tumor samples}

The principle of targeted therapies is based on the selection of patients with the correct profile. The identification of the tumor phenotype/genotype must be a prerequisite to allowing the evaluation of new molecules in the appropriate subgroup. Therefore, the potential of personalized medicine in OCA depends on the systematic acquisition of quality tumor samples that allow detailed molecular analysis using high-performance technologies. The highly variable quality of archived paraffin samples limits their translation value and has undoubtedly contributed to conflicting data in biomarker studies in the literature. Validation of predictive markers and prognoses will probably require the acquisition of frozen tumor samples.

Finally, given the capacity for tumor adaptation, the molecular image of an ovarian tumor is likely to change significantly between diagnosis and recurrence. It is essential that trials of new molecules in advanced stages be accompanied by complementary biological studies based on the tumor profile at relapse. More and more phase I trials request an optional metastatic biopsy and few patients refuse.

\section{Liquid biopsies in OCAs}

One of the main challenges in translational research in OCA is access to sequential tumor samples. Recurrences are frequently intra-abdominal in the form of diffuse peritoneal carcinomatosis and a biopsy is often difficult to perform. This carcinomatosis is responsible for the formation of ascites, which is often necessary to drain iteratively to relieve patients. These punctures could provide access to tumor cell samples throughout the disease. Some groups have established the feasibility and usefulness of proteomic and genomic analyzes of ascites cells. For example, Carden et al. have successfully evaluated the activation of the PI3K pathway in ascites cells by detecting mutations, amplifications and deletions of PIK3CA or PTEN (27). Another easily available "liquid biopsy" option is circulating tumor cells (CTC). Recent data in breast or prostate cancer and, more recently, OCA attest to the 
possible prognostic, even predictive, value of CTC (28). In the trial that evaluated temsirolimus, CTC was detected in $45 \%$ of patients with recurrent OCA and the presence of CTC correlated with the risk of progression (19). Another strategy is the study of circulating DNA. Rare activating mutations have been identified by sequencing circulating tumor DNA in OCAs (29).

\section{New testing models}

Modifications to the traditional phase I/II model could be proposed to make the tests more suitable for the biology of OCAs. Given the impressive molecular diversity of OCAs, the design of clinical trials aimed at a potentially identifiable single disorder in less than $10 \%$ of patients runs the risk of generating an exponential number of clinical studies that recruit very few patients. New prototypes of clinical trials that allow the inclusion of several molecules or the inclusion of several primitives with a common genomic portrait can be proposed. OCAs also have various tumor evolutions. It would be difficult to interpret a clinical trial evaluating a Mek inhibitor in mutated or amplified KRAS carcinomas, including low-grade type I and high-grade type II tumors. The random discontinuation design could be a suggested model. After an initial treatment phase, patients showing stable disease are randomized to placebo and continued treatment (30). This scheme is particularly suitable for certain type I carcinomas that are often not very progressive when this scheme makes it possible to determine the actual contribution of treatment to disease control.

\section{Conclusions}

The crucial information obtained in the last 10 years about the biology of OCAs has highlighted the real potential of a therapeutic approach adapted to the molecular profile. To allow these advances to lead to specific clinical applications, it is imperative that clinical research evaluating the efficacy of new molecules take into account the heterogeneity and plasticity of OCAs. It is essential that the trials recruit the appropriate patients, which requires the systematic acquisition of quality samples to allow a complete analysis of the genomic and potentially proteomic abnormalities of each tumor. Given the tumor heterogeneity of OCAs, there is little doubt that sensitivity to targeted treatment will require the expression or activation of the targeted oncogene and the demonstration of dependence on this oncogene (oncogene addiction). It will be necessary to adopt new models of clinical trials that incorporate auxiliary biological tests to promote the development of new molecules in OCA. In particular, obtaining sequential tumor samples (post-treatment or relapse) seems crucial. It is likely that for type I carcinomas, typically chemoresistant, targeted treatments can have a crutial role in the front line. For type II carcinomas, typically very chemosensitive but whose relapse is virtually unavoidable, the incorporation of new molecules into treatment would undoubtedly be more appropriate after chemotherapy to treat residual resistant clones.

\section{Acknowledgments}

Funding: None.

\section{Footnote}

Provenance and Peer Review: This article was commissioned by the Guest Editor (Heriberto Medina-Franco) for the series "Ovarian Cancer" published in Chinese Clinical Oncology. The article was sent for external peer review organized by the Guest Editor and the editorial office.

Conflicts of Interest: The author has completed the ICMJE uniform disclosure form (available at http://dx.doi. org/10.21037/cco-20-31). The series "Ovarian Cancer" was commissioned by the editorial office without any funding or sponsorship. The author has no other conflicts of interest to declare.

Ethical Statement: The author is accountable for all aspects of the work in ensuring that questions related to the accuracy or integrity of any part of the work are appropriately investigated and resolved.

Open Access Statement: This is an Open Access article distributed in accordance with the Creative Commons Attribution-NonCommercial-NoDerivs 4.0 International License (CC BY-NC-ND 4.0), which permits the noncommercial replication and distribution of the article with the strict proviso that no changes or edits are made and the original work is properly cited (including links to both the formal publication through the relevant DOI and the license). See: https://creativecommons.org/licenses/by-nc-nd/4.0/. 


\section{References}

1. de La Motte Rouge T, Petrella MC, Michels J, et al. New drugs and targeted therapeutic agents in ovarian cancer. Bull Cancer 2009;96:1215-24.

2. Kurman RJ, Shih IeM. Molecular pathogenesis and extraovarian origin of epithelial ovarian cancer--shifting the paradigm. Hum Pathol 2011;42:918-31.

3. Dann RB, DeLoia JA, Timms KM, et al. BRCA1/2 mutations and expression: response to platinum chemotherapy in patients with advanced stage epithelial ovarian cancer. Gynecol Oncol 2012;125:677-82.

4. Carden CP, Yap TA, Kaye SB. PARP inhibition: targeting the Achilles' heel of DNA repair to treat germline and sporadic ovarian cancers. Curr Opin Oncol 2010;22:473-80.

5. Zorn KK, Bonome T, Gangi L, et al. Gene expression profiles of serous, endometrioid, and clear cell subtypes of ovarian and endometrial cancer. Clin Cancer Res 2005;11:6422-30.

6. Cancer Genome Atlas Research Network. Integrated genomic analyses of ovarian carcinoma. Nature 2011;474:609-15. Erratum in: Nature. 2012 Oct 11;490(7419):298.

7. Matulonis UA, Hirsch M, Palescandolo E, et al. High throughput interrogation of somatic mutations in high grade serous cancer of the ovary. PLoS One 2011;6:e24433.

8. Sales E, Penson RT, Sullivan LA, et al. A snapshot of personalized care: molecular diagnostics in gynecologic cancer. J Clin Oncol 2012;30S:abstract 5029.

9. Wong KK, Tsang YT, Deavers MT, et al. BRAF mutation is rare in advanced-stage low-grade ovarian serous carcinomas. Am J Pathol 2010;177:1611-7.

10. Wang SE, Narasanna A, Perez-Torres M, et al. HER2 kinase domain mutation results in constitutive phosphorylation and activation of HER2 and EGFR and resistance to EGFR tyrosine kinase inhibitors. Cancer Cell 2006;10:25-38.

11. Jones S, Wang TL, Shih IeM, et al. Frequent mutations of chromatin remodeling gene ARID1A in ovarian clear cell carcinoma. Science 2010;330:228-31.

12. Anglesio MS, George J, Kulbe H, et al. IL6-STAT3-HIF signaling and therapeutic response to the angiogenesis inhibitor sunitinib in ovarian clear cell cancer. Clin Cancer Res 2011;17:2538-48.

13. Tan DS, Iravani M, McCluggage WG, et al. Genomic analysis reveals the molecular heterogeneity of ovarian clear cell carcinomas. Clin Cancer Res 2011;17:1521-34.

14. Cho KR, Shih IeM. Ovarian cancer. Annu Rev Pathol 2009;4:287-313.

15. Kelemen LE, Kobel M. Mucinous carcinomas of the ovary and colorectum: different organ, same dilemma. Lancet Oncol 2011;12:1071-80.

16. Olaussen KA, Dunant A, Fouret P, et al. DNA repair by ERCC1 in non-small-cell lung cancer and cisplatin-based adjuvant chemotherapy. N Engl J Med 2006;355:983-91.

17. Bouwman P, Jonkers J. The effects of deregulated DNA damage signalling on cancer chemotherapy response and resistance. Nat Rev Cancer 2012;12:587-98.

18. Leary A, Gore H. Incorporating bevacizumab into ovarian cancer treatment: practical considerations. Am Soc Clin Oncol Educ Book 2011;1:198-203.

19. Behbakht K, Sill MW, Darcy KM, et al. Phase II trial of the mTOR inhibitor, temsirolimus and evaluation of circulating tumor cells and tumor biomarkers in persistent and recurrent epithelial ovarian and primary peritoneal malignancies: a Gynecologic Oncology Group study. Gynecol Oncol 2011;123:19-26.

20. Janku F, Wheler JJ, Westin SN, et al. PI3K/AKT/ $\mathrm{mTORinhibitors} \mathrm{in} \mathrm{patients} \mathrm{with} \mathrm{breast} \mathrm{and} \mathrm{gynecologic}$ malignancies harboring PIK3CA mutations. J Clin Oncol 2012;30:777-82.

21. Di Nicolantonio F, Arena S, Tabernero J, et al. Deregulation of the PI3K and KRAS signaling pathways in human cancer cells determines their response to everolimus. J Clin Invest 2010;120:2858-66.

22. Buckanovich RJ, Berger R, Sella A, et al. Activity of cabozantinib (XL184) in advanced ovarian cancer patients (pts): Results from a phase II randomized discontinuation trial (RDT). J Clin Oncol 2011;29:abstract 5008.

23. Schilder RJ, Sill MW, Chen X, et al. Phase II study of gefitinib in patients with relapsed or persistent ovarian or primary peritoneal carcinoma and evaluation of epidermal growth factor receptor mutations and immunohistochemical expression: a Gynecologic Oncology Group study. Clin Cancer Res 2005;11:5539-48.

24. Makhija S, Amler LC, Glenn D, et al. Clinical activity of gemcitabine plus pertuzumab in platinum-resistant ovarian cancer, fallopian tube cancer, or primary peritoneal cancer. J Clin Oncol 2010;28:1215-23.

25. Westin SN, Herzog TJ, Coleman RL. Investigational agents in development for the treatment of ovarian cancer. Invest New Drugs 2013;31:213-29.

26. Chang HL, Pieretti-Vanmarcke R, Nicolaou F, et al. 
Mullerian inhibiting substance inhibits invasion and migration of epithelial cancer cell lines. Gynecol Oncol 2011;120:128-34.

27. Carden CP, Stewart A, Thavasu P, et al. The association of PI3 kinase signaling and chemoresistance in advanced ovarian cancer. Mol Cancer Ther 2012;11:1609-17.

28. Poveda A, Kaye SB, McCormack R, et al. Circulating tumor cells predict progression free survival and overall survival in patients with relapsed/recurrent advanced

Cite this article as: Lino-Silva LS. Ovarian carcinoma: pathology review with an emphasis in their molecular characteristics. Chin Clin Oncol 2020;9(4):45. doi: 10.21037/ cco-20-31 ovarian cancer. Gynecol Oncol 2011;122:567-72 .

29. Forshew T, Murtaza M, Parkinson C, et al. Noninvasive identification and monitoring of cancer mutations by targeted deep sequencing of plasma DNA. Sci Transl Med 2012;4:136ra68.

30. Penel N, Saleron J, Lansiaux A, et al. Methodological approaches of clinical studies with targeted therapies. Bull Cancer 2008;95:185-90. 